\title{
Canadian
} Science Publishing

Canadian Journal of Physics

Revue canadienne de physique

\section{Strain and different edge terminations modulated electronic and magnetic properties of armchair AIN/SiC nanoribbons: first-principles study}

\begin{tabular}{|r|l|}
\hline Journal: & Canadian Journal of Physics \\
\hline Manuscript ID & Cjp-2017-0092.R1 \\
\hline Manuscript Type: & Article \\
\hline Date Submitted by the Author: & $17-$ May-2017 \\
\hline Complete List of Authors: & $\begin{array}{l}\text { Du, Xiu-Juan; Taiyuan University of Science and Technology } \\
\text { Zhang, Zheng-Wei; The Xinjiang Technical Institute of Physics \& Chemistry } \\
\text { Song, Yu-ling; Nanyang Normal University, College of Physics and } \\
\text { Electronic Engineering }\end{array}$ \\
\hline Keyword: & $\begin{array}{l}\text { AlN/SiC nanoribbons, the strain, edge terminations, electronic and } \\
\text { magnetic properties, first-principles }\end{array}$ \\
\hline $\begin{array}{r}\text { Is the invited manuscript for } \\
\text { consideration in a Special } \\
\text { Issue? : }\end{array}$ & N/A \\
\hline
\end{tabular}

\section{SCHOLARONE \\ Manuscripts}




\title{
Strain and different edge terminations modulated electronic and magnetic properties of armchair AIN/SiC nanoribbons: first-principles study
}

\author{
Xiu-Juan Du, Zheng-Wei Zhang, and Yu-Ling Song
}

\begin{abstract}
Using first-principle calculations based on density functional theory, we investigate the strain and different edge terminations modulated electronic and magnetic properties of armchair AlN/SiC nanoribbons. The results show that the edge terminations $\mathrm{Fe}, \mathrm{Co}, \mathrm{Cl}$ can decrease or even eliminate the edge deformation of AlN/SiC nanoribbon. The magnetism of the nanoribbons is greatly adjusted by magnetic atoms $\mathrm{Fe}$ and $\mathrm{Co}$, but not by $\mathrm{Cl}$ atoms. Apart from the nanoribbon with $\mathrm{Cl}$ terminations, the magnetism of the residual nanoribbons can be adjusted by increasing the compressed or stretched strain.

The magnetic semiconductor nanoribbon with Co terminations becomes a magnetic half-metal system and then becomes a magnetic metal system, with the increase of the compressed strain. The magnetism of the nanoribbon with dangling bonds is attributed to the $\mathrm{SiC}$ edge and its nearest-neighbour $\mathrm{C}$ atoms, whereas the magnetism of the nanoribbon with $\mathrm{Fe}$ (or $\mathrm{Co}$ ) terminations is mainly contributed by Fe (or Co) terminations.
\end{abstract}

Keywords: AIN/SiC nanoribbons, the strain, edge terminations, electronic and magnetic properties, first-principles.

PACS Nos.: $\quad$ 73.21.-b, 73.22.-f.

\section{Introduction}

Graphene nanoribbons (GNRs) have received ever-increasing attention because of their successful fabrication [1-4], unique electronic properties and potential applications in spintronic device [5-7]. The excellent properties of GNRs greatly encourage researcher to find similar material. So far, pure AlN and SiC

X.-J. Du. School of Applied Science, Taiyuan University of Science and Technology, Taiyuan 030024, Shanxi, PR China.

Z.-W. Zhang. The key Laboratory of Plant Resources and Chemistry of Arid Zones, The Xinjiang Technical Institute of Physics \& Chemistry, Chinese Academy of Sciences, Urumchi 830011, Xinjiang, PR China.

Y.-L. Song. College of Physics and Electronic Engineering, Nanyang Normal University, Nanyang 473061, Henan, PR China.

Corresponding author: Xiu-Juan Du (duxiujuan@tyust.edu.cn). 
nanoribbons (NRs) have been widely studied due to their high thermal stability and potential technology applications.

In experiment, $\mathrm{AlN}[8,9]$ and $\mathrm{SiC}[10,11] \mathrm{NRs}$ have been successfully synthesized. Wu et al. [8] synthesized AlN NRs by evaporating $\mathrm{Al}$ powder in $\mathrm{NH}_{3} / \mathrm{N}_{2}$ atmosphere at $1200{ }^{\circ} \mathrm{C}$. Xie et al. [9] obtained the serrated AlN NRs by chloride assisted vapor-solid route. Wu et al. [10] synthesized SiC NRs by a reaction of $\mathrm{Si}$ and $\mathrm{CNTs}$ without catalysts using the simple evaporation method. Zhang et al. [11] synthesized SiC NRs by exposing Si powder and carbon black powder placed in a horizontal tube furnace to temperatures ranging from $1250{ }^{\circ} \mathrm{C}$ to $1500{ }^{\circ} \mathrm{C}$ for $5-12 \mathrm{~h}$ in an $\mathrm{Ar}$ atmosphere at atmospheric pressure. In theory, the effects of vacancy defect [12], doping [13], unpassivated edges of NRs [14], spin state [15] on the geometric structures and electronic properties of pristine AlN or SiC NRs have been also studied.

To meet the various demands for high performance electronic devices, the electronic structures and magnetic properties of NRs can be modulated by changing edge terminations of NRs and constituting heterogeneous NRs by the interweaving of AlN and SiC NRs (denoted by AlN/SiC NRs). Such a heterostructure is chosen partly because of similar lattice constants $(3.09$ and $3.07 \AA$ for $\mathrm{AlN}$ and $\mathrm{SiC}$, respectively) [16]. The important effect of dangling bond on the electronic and magnetic properties of AlN/SiC NRs was verified by our previous works [17,18]. In addition, transition-metal Fe and Co atoms have been used to decorate the edges of $\mathrm{BN}, \mathrm{SiC}$ and graphene NRs [19-21], and halogen $\mathrm{F}, \mathrm{Cl}$ atoms have been also used to modulate the electronic and magnetic properties of AlN and SiC NRs [22,23]. Therefore, in the present work, we will further investigate external strain and different edge terminations $(\mathrm{Fe}, \mathrm{Co}$ and $\mathrm{Cl}$ atoms) modulated electronic and magnetic properties of armchair AlN/SiC NRs within the density functional theory (DFT).

\section{Calculation method and models}

The DFT calculations are performed using the Vienna Ab-initio Simulation Package (VASP) [24-28], with the projected augmented wave (PAW) potentials [29] for the electron-ionic core interactions. The electron exchange-correlation is treated by the Perdew-Burke-Ernzerhof (PBE) formulation [30] of the generalized-gradient approximation (GGA), which yields the correct ground-state structure of the systems. 
The cut-off energy of the plane waves is $450 \mathrm{eV}$. The valence electrons throughout work are respectively Al- $3 s^{2} 3 p, N-2 s^{2} 2 p^{3}, S i-3 s^{2} 3 p^{2}$ and C- $-2 s^{2} 2 p^{2}$. The Brillouin zones (BZ) integration is performed within a Gamma centered Monkhorst-Pack scheme [31] using $1 \times 1 \times 5$ and $1 \times 1 \times 11$ k-point meshes for geometric optimization and further calculations of electronic properties, respectively. The 20 k-points along high symmetry direction are used to calculate the accurate band structure. The Gaussian smearing broadening is chosen as $0.2 \mathrm{eV}$. The energies and the forces on each ion are respectively converged to less than $10^{-4} \mathrm{eV} /$ atom and $0.02 \mathrm{eV} / \AA$.

The width of NR is defined as the total number of the dimer lines $\left(\mathrm{N}_{\mathrm{a}}\right)$ across the ribbon width. In the present work, in order to make AlN/SiC NRs have a geometric symmetry and ignore the effects of different AlN content on the electronic and magnetic properties of AlN/SiC NRs, we firstly cleaved a two-atom layer AlN sheet from the bulk wurtzite AlN crystal, and secondly cleaved an armchair AlN NR with the width $\mathrm{N}_{\mathrm{a}}$ $=11$ from the AlN sheet, then replaced five Al-N dimer lines of the cleaved AlN NR with Si-C dimer lines, and thus the special armchair AlN/SiC NR containing almost a half AlN and half SiC is obtained. Such a hybrid system is not still observed in experiments, but the analogous models have been studied [32,33]. Our study can offer important theory data for the future experimental researches of monolayer AlN/SiC nanoribbon. Fig. 1 depicts the top (left) and side (right) views of periodic boundary model of the obtained armchair AlN/SiC NR with edge dangling bonds. The original AlN/SiC NR is a two-atom layer NR periodically extended along the $\mathrm{Z}$ direction and assumed as infinite length to avoid end effects. To avoid the interaction between the neighboring ribbons, the NRs are separated from each other by the vacuum region with $16 \AA$ in both $\mathrm{X}$ and $\mathrm{Y}$ directions.

Fig. 1. The top (left) and side (right) views of periodic boundary model of the armchair AlN/SiC NR with edge dangling bonds. The number $\mathrm{N}_{\mathrm{a}}$ stands for the width of the ribbon. The red-dash-line rectangle contains an original periodic unit cell. The orange, grey, blue and pink balls represent the $\mathrm{Si}, \mathrm{C}, \mathrm{N}$ and $\mathrm{Al}$ atoms, respectively. 


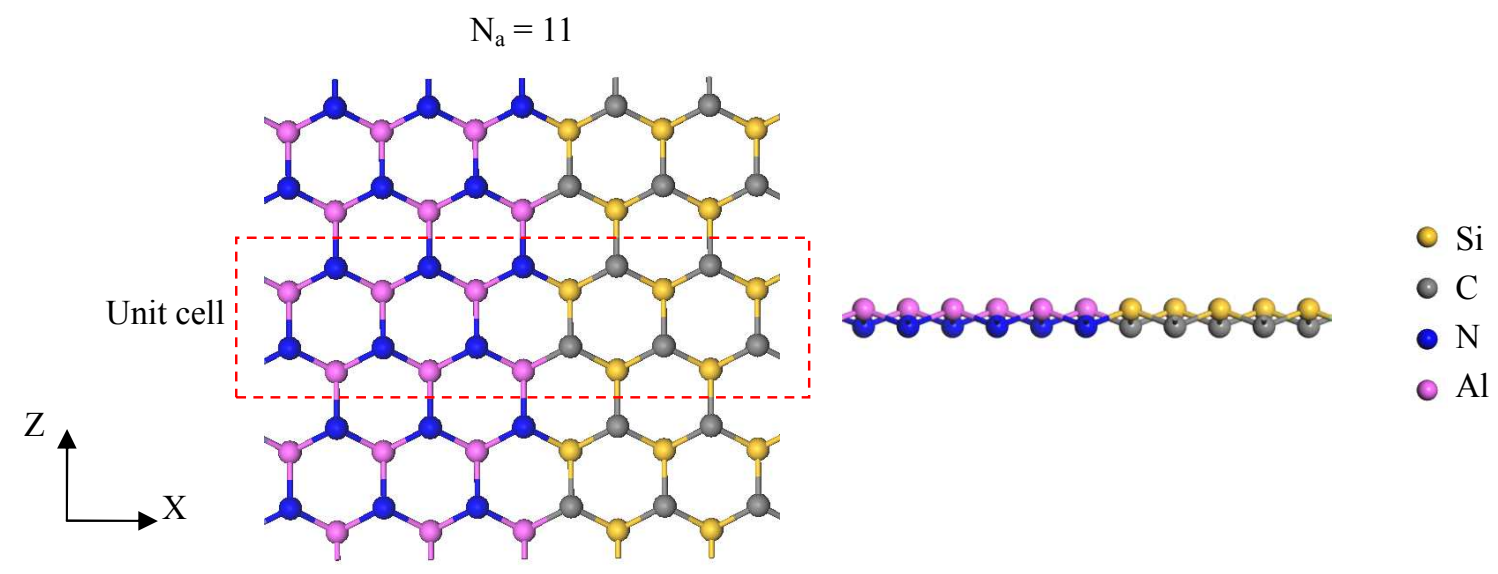

\section{Results and discussions}

\subsection{The optimized geometry, the strengths of edge bonds and interface bonds}

In this work, the $\mathrm{Fe}, \mathrm{Co}$ and $\mathrm{Cl}$ atoms are respectively chosen as edge terminations to investigate the effects of edge terminations on the magnetism and electronic properties of NRs. Figure 2 shows the top (left) and side (right) views of the optimized unit cell geometry of armchair AlN/SiC NRs with (a) edge dangling bonds, (b) Fe terminations, (c) Co terminations and (d) $\mathrm{Cl}$ terminations. Compared with the Fig. 1, the four optimized AlN/SiC NRs in Fig. 2 basically present a single-atom layer. The edge terminations ( $\mathrm{Fe}, \mathrm{Co}, \mathrm{Cl}$ ) and the corresponding AlN/SiC NR are not in the same plane. From the Fig. 2a, it can be seen that the shorter bond lengths of the Al-N (1.720 $\AA)$ and Si-C bonds (1.702 $\AA)$ located respectively at the left and right edge of NR indicates that theses two bonds are stronger. In addition, the edge structure of NR presents a larger deformation. This is because that the vanishing of the neighbor atoms outside the NR after being cleaved from bulk crystal not only affects the distribution of charge density but also causes the structure relaxation. Figures $2 \mathrm{~b}-2 \mathrm{~d}$ indicate that the edge terminations $\mathrm{Fe}, \mathrm{Co}, \mathrm{Cl}$ can decrease or even eliminate the edge deformation of AlN/SiC NR.

In Figs. 2b-2d, the bond lengths of the edge bonds causing by the terminated atoms (i.e., $\mathrm{Fe}, \mathrm{Co}$ and $\mathrm{Cl}$ atoms) and interface bonds causing by the heterogeneous interface of the AlN/SiC NR are labeled at an appropriate location. To conveniently analyze the bond strengths of the edge bonds and the interface bonds, Table 1 further shows a list of the bond length of edge bonds and interface bonds of the optimized AlN/SiC NRs with different edge terminations ( $\mathrm{Fe}$ or $\mathrm{Co}$ or $\mathrm{Cl}$ terminations). As illustrated in Table 1, it can be seen that for the AlN/SiC NR with Fe (or Co) terminations, the length of the four edge bonds Fe-Al, Fe-Si, Fe-C 
and Fe-N (or Co-Al, Co-Si, Co-C and Co-N) successively decrease and thus the bond strengths successively increase. However, when the edge terminations are $\mathrm{Cl}$ atoms, the rule above is false, we can find that $\mathrm{Cl}-\mathrm{C}$ $(1.741 \AA)$ and Cl-N (1.743 $\AA)$ bonds have a small bond-length difference. This is because that Fe and Co atoms are transition-metal atoms with a similar electronegativity ( $\mathrm{Fe}(1.83)$ and $\mathrm{Co}(1.88))$, whereas the $\mathrm{Cl}$ atom is the halogen atom with a larger electronegativity $(\mathrm{Cl}(3.16))$. For these three NRs (shown in Figs. 2b-2d), Table 1 displays the Al-C interface bond is weaker than the Si-N interface bond of the same system. Interestingly, the four edge bonds $\mathrm{Cl}-\mathrm{Al}, \mathrm{Cl}-\mathrm{Si}, \mathrm{Cl}-\mathrm{C}$ and $\mathrm{Cl}-\mathrm{N}$ of $\mathrm{AlN} / \mathrm{SiC} \mathrm{NR}$ with $\mathrm{Cl}$ terminations are stronger than the corresponding edge bonds of the residual two NRs (Figs. 2b and 2c), whereas the interface bonds $\mathrm{Al}-\mathrm{C}$ and $\mathrm{Si}-\mathrm{N}$ of $\mathrm{NR}$ with $\mathrm{Cl}$ terminations (Fig. 2d) are weaker than the corresponding interface bonds of the residual two NRs (Figs. $2 b$ and $2 c$ ).

Fig. 2. The top (left) and side (right) views of the optimized unit cell geometry of armchair AlN/SiC NRs with (a) edge dangling bonds, $(b)$ Fe terminations, $(c)$ Co terminations and $(d) \mathrm{Cl}$ terminations.

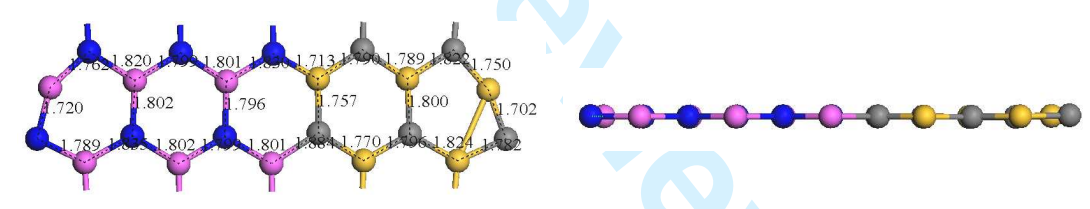

(a) AlN/SiC NR with edge dangling bonds

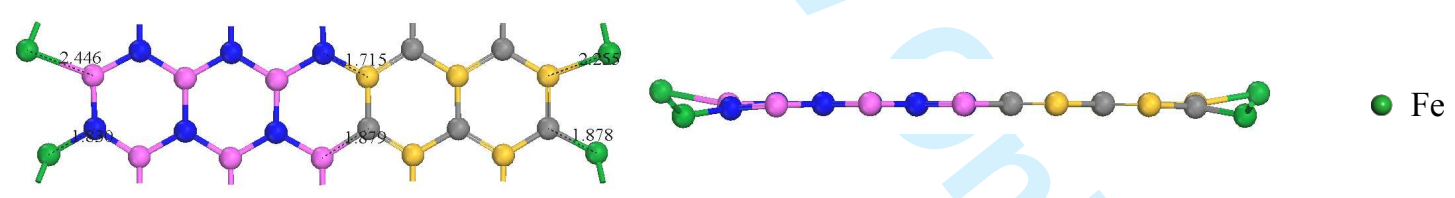

(b) AlN/SiC NR with Fe terminations

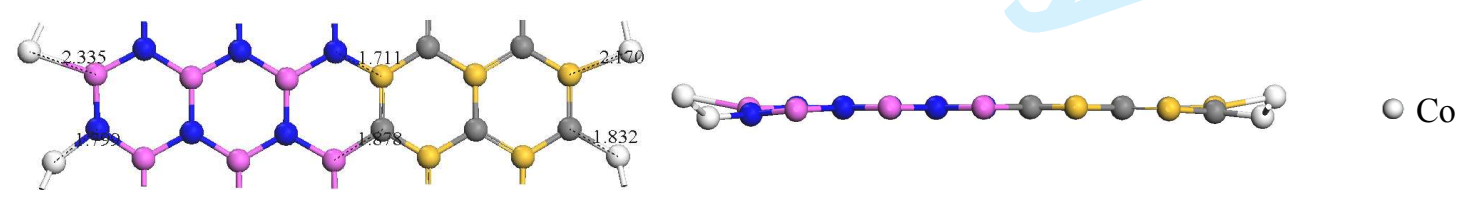

(c) AlN/SiC NR with Co terminations
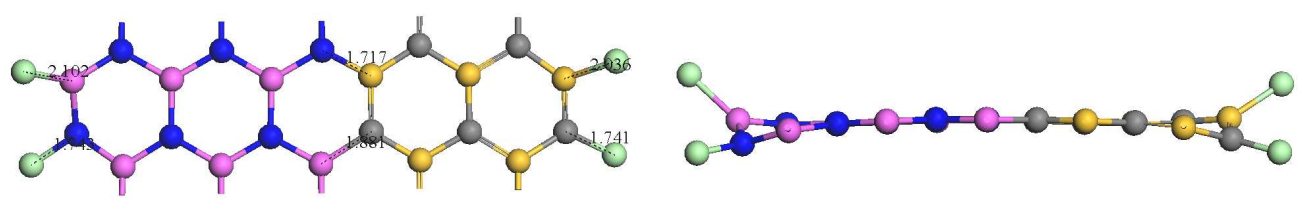

$\mathrm{Cl}$

(d) AlN/SiC NR with $\mathrm{Cl}$ terminations 
Table 1. The bond length of edge bonds and interface bonds of the optimized AlN/SiC NRs with different edge terminations (i.e. Fe or Co or $\mathrm{Cl}$ terminations).

\begin{tabular}{|c|c|c|c|c|c|}
\hline \multicolumn{2}{|c|}{ AlN/SiC NR with Fe terminations } & \multicolumn{2}{|c|}{ AlN/SiC NR with Co terminations } & \multicolumn{2}{|c|}{ AlN/SiC NR with $\mathrm{Cl}$ terminations } \\
\hline Type of the bonds & Bond length $(\AA)$ & Type of the bonds & Bond length $(\AA)$ & Type of the bonds & Bond length $(\AA)$ \\
\hline $\mathrm{Fe}-\mathrm{Al}$ & 2.446 & Co-Al & 2.335 & $\mathrm{Cl}-\mathrm{Al}$ & 2.102 \\
\hline $\mathrm{Fe}-\mathrm{Si}$ & 2.255 & $\mathrm{Co}-\mathrm{Si}$ & 2.170 & $\mathrm{Cl}-\mathrm{Si}$ & 2.036 \\
\hline $\mathrm{Fe}-\mathrm{C}$ & 1.878 & $\mathrm{Co}-\mathrm{C}$ & 1.832 & $\mathrm{Cl}-\mathrm{C}$ & 1.741 \\
\hline Fe-N & 1.830 & $\mathrm{Co}-\mathrm{N}$ & 1.799 & Cl-N & 1.743 \\
\hline Al-C & 1.879 & Al-C & 1.878 & Al-C & 1.881 \\
\hline Si-N & 1.715 & Si-N & 1.711 & $\mathrm{Si}-\mathrm{N}$ & 1.717 \\
\hline
\end{tabular}

\subsection{The binding energy}

In order to investigate the structural stabilities of the AlN/SiC NRs with different edge terminations, we calculated the binding energies of five systems in Table 2 according to

$$
\Delta E=E_{\text {tot }}-E_{\text {dangling }}-l E_{F e}-m E_{C o}-n E_{C l}
$$

where $E_{\text {tot }}, E_{\text {dangling }}, E_{F e}, E_{C o}$ and $E_{C l}$ are respectively the total energy of the investigated system, the total energy of AlN/SiC NR with edge dangling bonds, the total energy of isolated $\mathrm{Fe}, \mathrm{Co}$ and $\mathrm{Cl}$ atoms; $l$, $m$ and $n$ are the number of $\mathrm{Fe}, \mathrm{Co}$ and $\mathrm{Cl}$ atoms, respectively.

From the Table 2, it can be seen that AlN/SiC NR with Fe or Co terminations (Fig. 2b or 2c) has a positive binding energy, implying the formation process of these two systems are endothermic and unstable; whereas the negative binding energy of $\mathrm{AlN} / \mathrm{SiC} \mathrm{NR}$ with $\mathrm{Cl}$ terminations (Fig. 2d) indicates that the corresponding passivation process is completely exothermic, and thus the system can be formed easily and exist steadily. Moreover, preliminary calculation in our next work shows that when two Fe or Co atoms of $\mathrm{AlN} / \mathrm{SiC} \mathrm{NR}$ with $\mathrm{Fe}$ or Co terminations (Fig. $2 \mathrm{~b}$ or $2 \mathrm{c}$ ) are replaced with two $\mathrm{Cl}$ atoms, the obtained $\mathrm{AlN} / \mathrm{SiC} \mathrm{NR}$ with two $\mathrm{Cl}$ and two $\mathrm{Fe}$ (or $\mathrm{Co}$ ) terminations (Fig .3a or 3b) has a negative binding energy and 
thus can exist steadily. These calculations can offer an effective approach to modulate the electronic and magnetic properties of $\mathrm{AlN} / \mathrm{SiC} \mathrm{NRs}$ for the future experimental researches.

Table 2. The calculated binding energy (eV) for AlN/SiC NRs with different edge terminations.

\begin{tabular}{cc}
\hline The systems & The binding energy (eV) \\
\hline AlN/SiC NR with Fe terminations (Fig. 2b) & 5.442 \\
\hline AlN/SiC NR with Co terminations (Fig. 2c) & 5.776 \\
\hline AlN/SiC NR with Cl terminations (Fig. 2d) & -8.550 \\
\hline AlN/SiC NR with two Cl and two Fe terminations (Fig. 3a) & -4.540 \\
\hline AlN/SiC NR with two Cl and two Co terminations (Fig. 3b) & -3.653 \\
\hline
\end{tabular}

Fig. 3. The top (left) and side (right) views of the optimized unit cell geometry of armchair AlN/SiC NRs with $(a)$ two Cl and two Fe terminations and $(b)$ two $\mathrm{Cl}$ and two Co terminations.

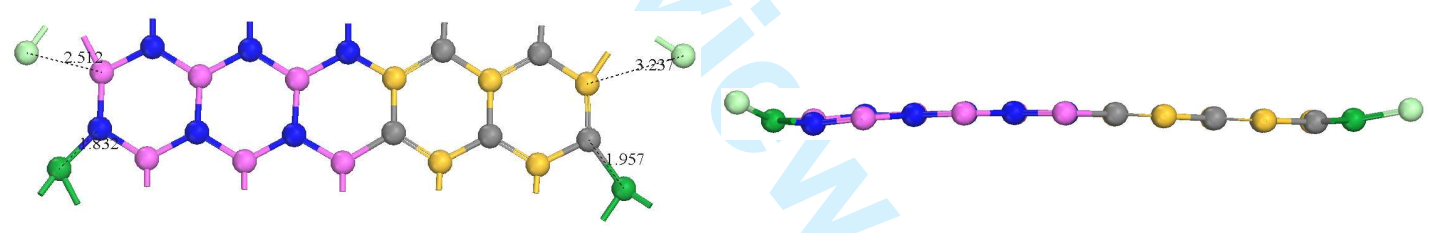

(a) AlN/SiC NR with two $\mathrm{Cl}$ and two Fe terminations
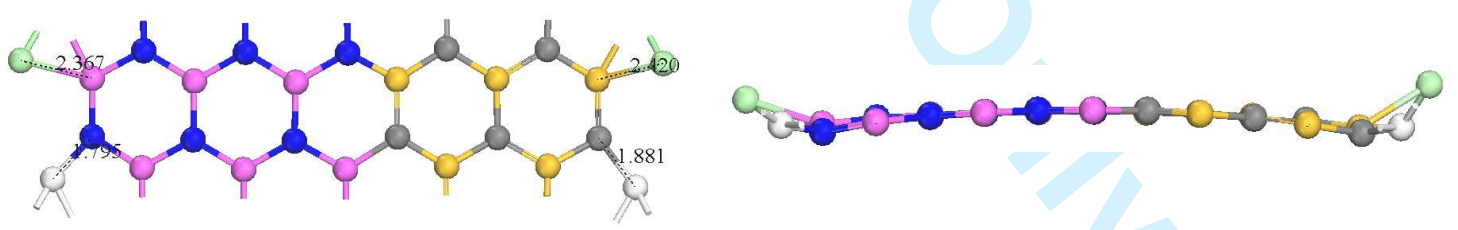

(b) AlN/SiC NR with two $\mathrm{Cl}$ and two Co terminations

\subsection{The band gap, magnetic moment and difference charge density}

To investigate the effect of the strain along the axis of ribbon on the total magnetic moment and band gap of AlN/SiC NRs, Fig. 4 shows band gaps and magnetic moments of the optimized AlN/SiC NRs with (a) edge dangling bonds, (b) Fe terminations, (c) Co terminations and (d) $\mathrm{Cl}$ terminations as a function of strain along the axis of the ribbon. The strain is calculated according to the formula $\varepsilon=\left(c-c_{0}\right) / c_{0} \times 100 \%$, where $c_{0}$ and $c$ are respectively the original length and compressed (or stretched) length of the unit cell along 
the axis of the ribbon. Positive (+) and minus (-) denotes the stretched strain and compressed strain, respectively. From four graphs shown in Fig. 4, we find that the magnetic moments of AlN/SiC NRs with (a) edge dangling bonds, (b) Fe terminations, (c) Co terminations and (d) $\mathrm{Cl}$ terminations are respectively 0 , 11.94, 7.88 and $0 \mu_{B}$ when the $\varepsilon=0$, indicating that the magnetism of the NRs is greatly adjusted by magnetic atoms $\mathrm{Fe}$ and $\mathrm{Co}$, but not by $\mathrm{Cl}$ atoms. Furthermore, the Figs. 4a-4c present that the strain can change the magnetism of NRs with edge dangling bonds (or Fe terminations or Co terminations). However, Fig. 4d reflects that the non-magnetic NRs with $\mathrm{Cl}$ terminations cannot become a magnetic system by applying strain. More specifically, the black and red curves shown in the Fig. 4a illustrates that non-magnetic semiconductor AlN/SiC NR with edge dangling bonds under $0 \%$ strain becomes a magnetic semiconductor systems when the compressive strain is $-4 \%$ and the stretched strain is $4 \%, 6 \%$ and $8 \%$. It is worth noting that the magnetic semiconductor NR shown in Fig. $4 \mathrm{~b}$ becomes a magnetic half-metal due to the two band gaps $0.24 \mathrm{eV}$ (black curve) and $0 \mathrm{eV}$ (red curve) as the compressive strain is $-6 \%$. Interestingly, with the increase of the compressed strain, the magnetic semiconductor NR shown in Fig. 4c (under $0 \%$ or $-2 \%$ compressed strain) becomes a magnetic half-metal system (under $-4 \%$ or $-6 \%$ compressed strain) and then becomes a magnetic metal system (under $-8 \%$ compressed strain). Finally, the band gaps of the non-magnetic semiconductor NR shown in Fig. 4d decrease (or increase) gradually, with the increasing stretched (or compressed) strain.

The variations of band gaps and magnetism of aforementioned AlN/SiC NRs with strains can be illustrated as follows: the different strain can cause different crystal deformation, and thus the charge distribution and the net up-spin charge of the system will be changed, and then the band gaps of majority spin and minority spin as well as the total magnetic moment will be changed.

To investigate the distribution of the magnetism, as an example, the Fig. 5 shows the difference charge density isosurfaces of $0.005 \mathrm{e} / \AA^{3}\left(\rho^{u p}-\rho^{\text {down }}\right)$ for the optimized AlN/SiC NRs with (a) edge dangling bonds (under $-4 \%$ compressed strain), (b) Fe terminations (under $-8 \%$ compressed strain), (c) Co terminations 
(under $-8 \%$ compressed strain). For the system shown in Fig. 5a, it is obviously that the net up-spin charge mainly accumulates at the $\mathrm{SiC}$ edge and its nearest-neighbour $\mathrm{C}$ atoms, reflecting that the magnetic moment largely contributed by the $\mathrm{SiC}$ edge and its nearest-neighbour $\mathrm{C}$ atoms. The calculated total magnetic moment of Fig. 5a is $1.94 \mu_{B}$. However, the net up-spin charges of these two systems in Figs. 5b and 5c are mainly located at the termination Fe and Co atoms, respectively. Therefore, the total magnetic moments of these two systems $\left(11.91 \mu_{B}\right.$ for Fig. $5 \mathrm{~b}$ and $7.12 \mu_{B}$ for Fig. 5c) are mainly attributed to Fe and Co terminations, respectively.

Fig. 4. Band gaps and magnetic moments of the optimized AlN/SiC NRs with (a) edge dangling bonds, $(b)$ Fe terminations,

(c) Co terminations and $(d) \mathrm{Cl}$ terminations as a function of strain along the axis of the ribbon.

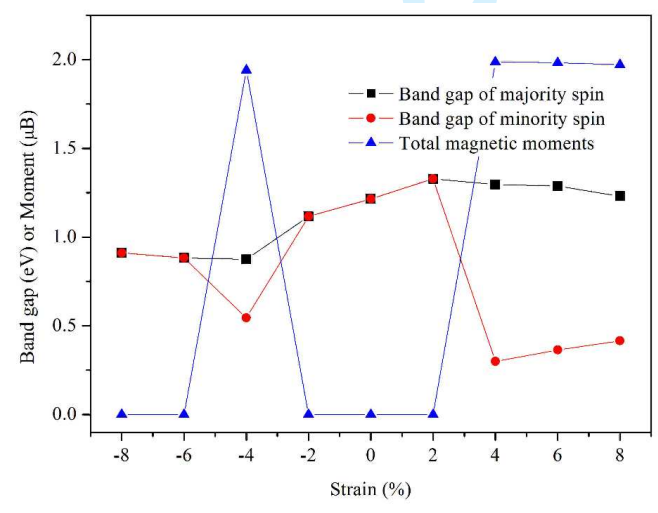

(a) AlN/SiC NR with edge dangling bonds

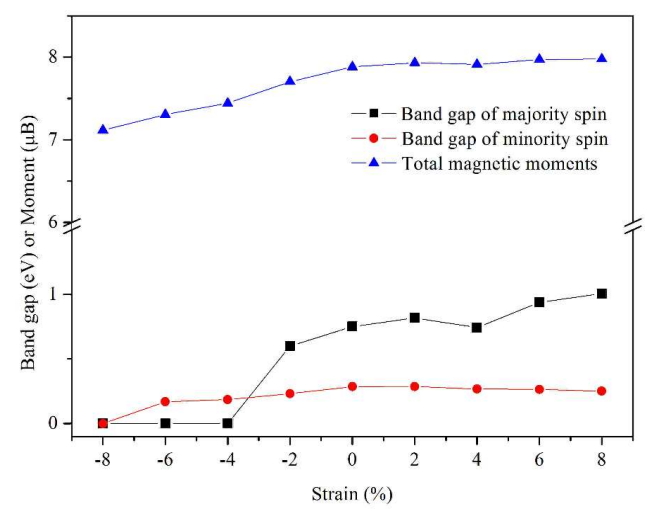

(c) AlN/SiC NR with Co terminations

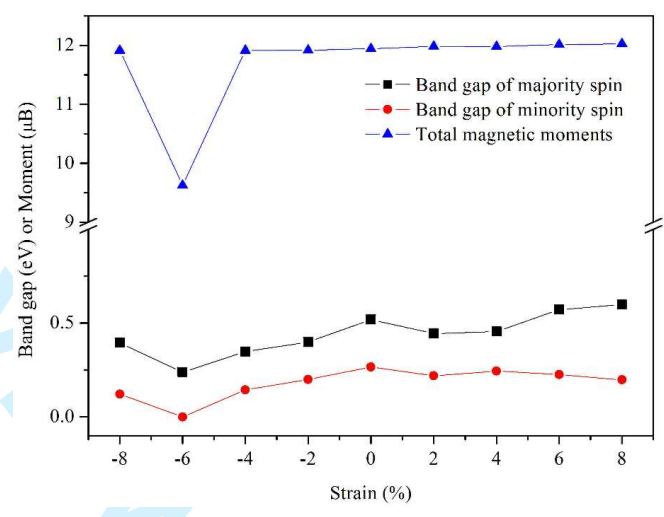

(b) AlN/SiC NR with Fe terminations

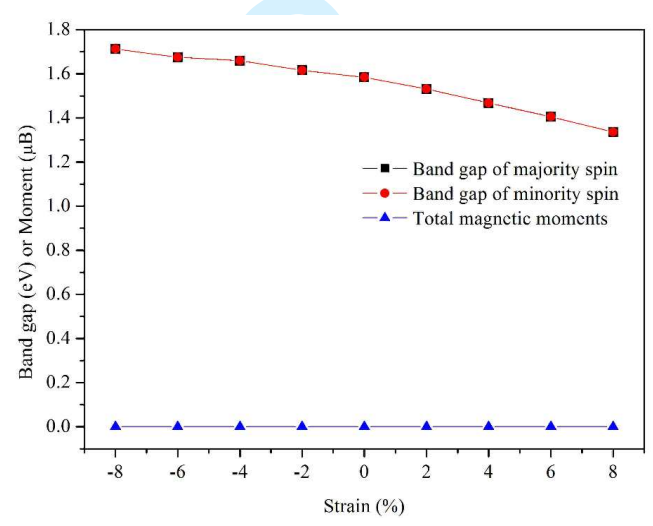

(d) AlN/SiC NR with $\mathrm{Cl}$ terminations

Fig. 5. The difference charge density isosurfaces of $0.005 \mathrm{e} / \AA^{3}\left(\rho^{u p}-\rho^{\text {down }}\right)$ for the AlN/SiC NRs with $(a)$ edge dangling bonds (under $-4 \%$ compressed strain), (b) Fe terminations (under $-8 \%$ compressed strain), (c) Co terminations (under $-8 \%$ compressed strain). 


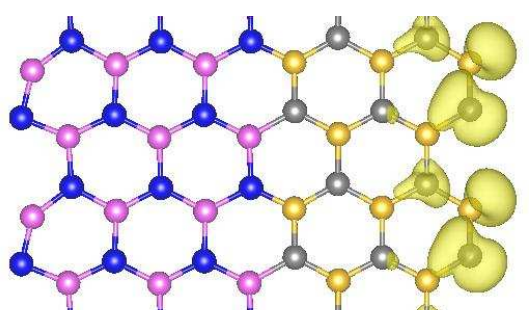

(a) AlN/SiC NR with edge dangling bonds

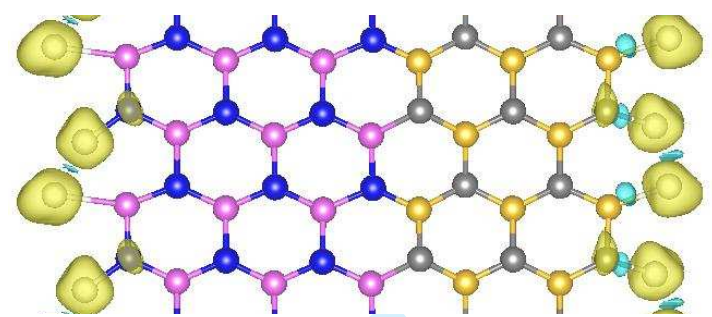

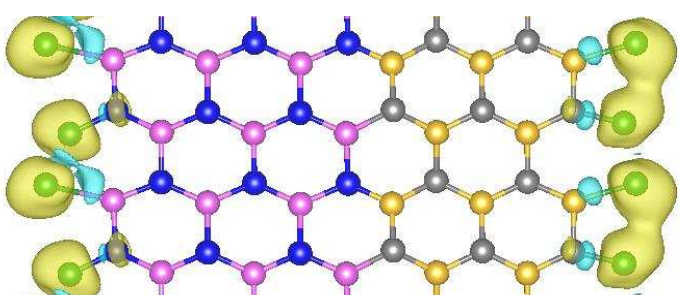

(b) AlN/SiC NR with Fe terminations

(c) AlN/SiC NR with Co terminations

\section{Conclusions}

In summary, external strain and different edge terminations modulated electronic and magnetic properties of armchair AlN/SiC NRs have been investigated within DFT. Following conclusions are drawn:

1. For AlN/SiC NR with edge dangling bonds, the left-edge Al-N (1.720 $\AA)$ and right-edge Si-C bonds $(1.702 \AA)$ are stronger and the edge structure of NR presents a larger deformation. The edge terminations $\mathrm{Fe}, \mathrm{Co}, \mathrm{Cl}$ can decrease or even eliminate the edge deformation of $\mathrm{AlN} / \mathrm{SiC} \mathrm{NR}$.

2. The four edge bonds $\mathrm{Cl}-\mathrm{Al}, \mathrm{Cl}-\mathrm{Si}, \mathrm{Cl}-\mathrm{C}$ and $\mathrm{Cl}-\mathrm{N}$ of $\mathrm{AlN} / \mathrm{SiC} \mathrm{NR}$ with $\mathrm{Cl}$ terminations are stronger than the corresponding edge bonds of NR with Fe or Co terminations, whereas the interface bonds Al-C and $\mathrm{Si}-\mathrm{N}$ of NR with $\mathrm{Cl}$ terminations are weaker than the corresponding interface bonds of NR with Fe or Co terminations.

3. The magnetism of the NRs is greatly adjusted by magnetic atoms $\mathrm{Fe}$ and $\mathrm{Co}$, but not by $\mathrm{Cl}$ atoms. The strain can change the magnetism of NRs with edge dangling bonds (or $\mathrm{Fe}$ terminations or $\mathrm{Co}$ terminations). The non-magnetic NRs with $\mathrm{Cl}$ terminations cannot become a magnetic system by applying strain. With the increase of the compressed strain, the magnetic semiconductor NR with Co terminations becomes a magnetic half-metal system and then becomes a magnetic metal system.

4. The magnetism of the NR with dangling bonds is attributed to the $\mathrm{SiC}$ edge and its nearest-neighbour $\mathrm{C}$ atoms, whereas the magnetism of the $\mathrm{NR}$ with $\mathrm{Fe}$ (or $\mathrm{Co}$ ) terminations is mainly contributed by $\mathrm{Fe}$ (or $\mathrm{Co}$ ) 
terminations.

\section{Acknowledgements}

The authors would like to acknowledge the support from the National Natural Science Foundation of China [grant number 11547211]; Doctoral Scientific Research Starting Foundation of Taiyuan University of Science and Technology [grant number 20152005]; the Director Foundation of XTIPC, CAS [grant number 2015KY005]; the Science and Technology Key Projects of Henan Province, China [grant number $1502102210124]$.

\section{References}

1. Y. Zhang, Y.W. Tan, H.L. Stormer, and P. Kim. Nature, 438, 201 (2005).

2. A.K. Geim, and K.S. Novoselov. Nature Mater. 6, 183 (2007).

3. C. Berger, Z.M. Song, X.B. Li, X.S. Wu, N. Brown, C. Naud, D. Mayou, T.B. Li, J. Hass, A.N. Marchenkov, E.H. Conrad, P.N. First, and W.A. de Heer. Science, 312, 1191 (2006).

4. K.S. Novoselov, A.K. Geim, S.V. Morozov, D. Jiang, M.I. Katsnelson, I.V. Grigorieva, S.V. Dubonos, and A.A. Firsov. Nature, 438, 197 (2005).

5. K.S. Novoselov, E. McCann, S.V. Morozov, V.I. Fal'ko, M.I. Katsnelson, U. Zeitler, D. Jiang, F. Schedin1, and A.K. Geim. Nat. Phys. 2, 177 (2006).

6. K. Andre Mkhoyan, Alexander W. Contryman, John Silcox, Derek A. Stewart, Goki Eda, Cecilia Mattevi, Steve Miller, and Manish Chhowalla. Nano Lett. 7, 1888 (2007).

7. B. Huang, Q.M. Yan, Z. Li, and W.H. Duan. Front. Phys. China 4, 269 (2009).

8. Q. Wu, Z. Hu, X.Z. Wang, Y. Chen, and Y.N. Lu. J. Phys. Chem. B 107, 9726 (2003).

9. T. Xie, Y. Lin, G.S. Wu, X.Y. Yuan, Z. Jiang, C.H. Ye, G.W. Meng, and L.D. Zhang. Inorg. Chem.Commun. 7, 545 (2004).

10. R. Wu, L. Wu, G. Yang, Y. Pan, J. Chen, R. Zhai, and J. Lin. J. Phys. D: Appl. Phys. 40, 3697 (2007).

11. H. Zhang, W. Ding, K. He, and M. Li. Nanoscale Res. Lett. 5, 1264 (2010).

12. F.L. Zheng, J.M. Zhang, Y. Zhang, and V. Ji. Physica B, 405, 3775 (2010).

13. Q.L. Rao, Y.X. Wang, Z. Chen, X.J. Du, and T.T. Sun. Superlattice. Microst. 84, 36 (2015).

14. M. Wu, X. Wu, Y. Pei, and X.C. Zeng. Nano Res. 4, 233 (2011).

15. Y. An, M. Zhang, L. Chen, C. Xia, T. Wang, Z. Fu, Z. Jiao, and G. Xu. RSC Advances, 5, 107136 (2015).

16. H. Şahin, S. Cahangirov, M. Topsakal, E. Bekaroglu, E. Akturk, R.T. Senger, and S. Ciraci. Phys. Rev. B 80, 155453 
(2009).

17. X.J. Du, Z. Chen, J. Zhang, Z.R. Ning, and X.L. Fan. J. Alloy. Compd. 586, 176 (2014).

18. T.t. Sun, Y.x. Wang, Z. Chen, and X.j. Du. Comp. Mater. Sci. 92, 372 (2014).

19. C. Cao, Y. Wang, H.-P. Cheng, and J.-Z. Jiang. Appl. Phys. Lett. 99, 073110 (2011).

20. Y. Wang, Y. Ding, and J. Ni. Appl. Phys. Lett. 99, 053123 (2011).

21. M. Wu, Y. Pei, and X.C. Zeng. Chem. Phys. Lett. 580, 78 (2013).

22. Y. Ding, and Y. Wang. J. Mater. Chem. C 4, 1517 (2016).

23. W. Chen, H. Zhang, X. Ding, G. Yu, D. Liu, and X. Huang. J. Mater. Chem. C 2, 7836 (2014).

24. G. Kresse, and J. Hafner. Phy. Rev. B 47, 558 (1993).

25. G. Kresse, and J. Hafner. Phy. Rev. B 49, 14251 (1994).

26. G. Kresse, and J. Furthmüller. Comput. Mater. Sci. 6, 15 (1996).

27. G. Kresse, and J. Furthmüller. Phy. Rev. B 54, 11169 (1996).

28. W. Kohn, and L.J. Sham. Phys. Rev. 140, A1133 (1965).

29. G. Kresse, and D. Joubert. Phy. Rev. B 59, 1758 (1999).

30. J.P. Perdew, K. Burke, and M. Ernzerhof. Phys. Rev. Lett. 77, 3865 (1996).

31. H.J. Monkhorst, and J.D. Pack. Phys. Rev. B 13, 5188 (1976).

32. S. Jun, X. Li, and F. Meng. Phys. Rev. B 83, 153407 (2011).

33. X. Li, C. Mi, F. Meng, and I.H. Lee. Comput. Mater. Sci. 78, 129 (2013). 